\title{
Diversidad y estructura del bosque de galería del río Fuerte, Sinaloa, México
}

\section{Diversity and structure of the gallery forest of the Fuerte river, Sinaloa, Mexico}

\section{Salvador \\ Maldonado ${ }^{1}$ : \\ Julián Darío Moreno-Aldaco ${ }^{2}$, \\ Estuardo Lara-Ponce 2,3 [] \\ Hugo Humberto Piña-Ruíz 2,3 \\ ${ }^{1}$ Laboratorio de Fisiología Vegetal. \\ Unidad de Biotecnología y Pro- \\ totipos. Facultad de Estudios \\ Superiores Iztacala. Universidad \\ Nacional Autónoma de México. \\ Los Reyes Iztacala, CP. 54090. \\ Tlalnepantla, Estado de México, \\ México. \\ ${ }^{2}$ Ingeniería Forestal. Unidad \\ Mochicahui. Universidad \\ Autónoma Indígena de México. \\ Mochicahui, CP. 81890 . El Fuerte, \\ Sinaloa, México. \\ ${ }^{3}$ Integrantes del Cuerpo \\ Académico Biodiversidad y \\ Estrategias Comunitarias de De- \\ sarrollo Sostenible. Mochicahui, \\ CP. 81890. El Fuerte, Sinaloa, \\ México. \\ *Autor de correspondencia: ssampayom@hotmail.com}

\section{Artículo científico}

Recibido: 30 de mayo 2021

Aceptado: 06 de agosto 2021

Como citar: Sampayo-

Maldonado S, Moreno-Aldaco

JD, Lara-Ponce E, Piña-Ruíz

HH (2021) Diversidad y estruc-

tura del bosque de galería del

río Fuerte, Sinaloa, México.

Ecosistemas y Recursos

Agropecuarios 8(2): e3046.

DOI: $10.19136 /$ era.a8n2.3046
RESUMEN. El río Fuerte es la corriente hidrológica más importante del norte de Sinaloa, su riqueza biológica forma parte del patrimonio biocultural del pueblo Yoreme-Mayo y es fundamental para su bienestar. Sin embargo, la contaminación, fragmentación y pérdida de biodiversidad están impactando de forma negativa su funcionalidad. Por lo anterior, el objetivo fue evaluar y describir la diversidad y la estructura de la vegetación asociada al bosque de galería del río Fuerte. Se establecieron 41 parcelas circulares de 0.1 ha, en las que se tomaron datos dasométricos (altura, diámetro normal y cobertura de la copa). La estructura se caracterizó mediante la distribución diamétrica y la altura, empleando el índice de valor de importancia. La diversidad se evaluó con los índices de Equidad $(E)$, Margalef $\left(D_{M g}\right)$, Simpson $(D)$ y Shannon-Wiener $\left(H^{\prime}\right)$. Se registraron 27 especies de las que 16 son nativas. La familia Fabaceae fue la más representada con 11 especies, seguida de Salicaceae con dos. El estrato arbóreo tuvo un valor de importancia del $79.18 \%$ y de $14.11 \%$ para el arbustivo. El $60 \%$ de los árboles se agrupó en la categoría diamétrica de 5 y el $74 \%$ en las categorías de 5 y 10 de altura. Las especies de mayor importancia ecológica para el ecosistema ribereño fueron Populus mexicana, Pithecellobium dulce y Prosopis juliflora. El río Fuerte presentó una diversidad alta con dominancia del estrato arbóreo y una población con estructura juvenil, característica de un ecosistema con presencia de disturbios por actividades humanas.

Palabras clave: Diversidad florística, ecología forestal, índice de valor de importancia, restauración, vegetación ribereña.

ABSTRACT. The Fuerte River is the most important hydrological current in northern Sinaloa, its biological wealth is part of the biocultural heritage of the Yoreme-Mayo people and is essential for their well-being. However, pollution, fragmentation and loss of biodiversity are negatively impacting its functionality. Therefore, the objective was to evaluate and describe the diversity and structure of the vegetation associated with the gallery forest of the Fuerte river. 41 circular plots of 0.1 ha were established, in which dasometric data (height, normal diameter and crown coverage) were taken. The structure was characterized by diameter distribution and height, using the importance value index. Diversity was evaluated with the Equity $(E)$, Margalef $\left(\mathrm{D}_{M g}\right)$, Simpson $(D)$ and Shannon-Wiener $\left(H^{\prime}\right)$ indices. 27 species were recorded of which 16 are native. The Fabaceae family was the most represented with 11 species, followed by Salicaceae with two. The arboreal stratum had an importance value of $79.18 \%$, and $14.11 \%$ for the shrub. $60 \%$ of the trees were grouped in the diameter category of 5 and $74 \%$ in the categories of 5 and 10 in height. The most ecologically important species for the riparian ecosystem were Populus mexicana, Pithecellobium dulce and Prosopis juliflora. The Fuerte river presented a high diversity with dominance of the arboreal stratum and a population with a youthful structure, characteristic of an ecosystem with the presence of disturbances due to human activities.

Key words: Floristic diversity, forest ecology, importance value index, restoration, riparian vegetation. 


\section{INTRODUCCIÓN}

El estado de Sinaloa tiene gran riqueza biológica, ya que el $60.2 \%$ de la superficie del estado tiene algún tipo de vegetación (INEGI 2014, SEMARNAT 2014). Al respecto Villaseñor y Ortiz (2014) reportaron para la entidad 2922 especies nativas de angiospermas, lo que representa el $13.37 \%$ del total del país. Mientras que el inventario forestal del estado registró 24 especies de gimnospermas, siendo el $13.11 \%$ de la riqueza de México (SEMARNAT 2014). No obstante la relevancia biológica, Monjardín-Armenta et al. (2017) advierten que Sinaloa pierde $126.5 \mathrm{~km}^{2}$ año ${ }^{-1}$ de vegetación, lo que significa una tasa media anual de deforestación del $0.41 \%$; lo que acelera la fragmentación de las poblaciones y la pérdida de la biodiversidad.

Los recursos forestales están ligados al desarrollo de las áreas culturales de México. Pero las actividades humanas como la agricultura, la ganadería, la minería y el incremento de la mancha urbana han transformado la distribución de la cubierta vegetal (Monjardín-Armenta et al. 2017). Al respecto Richardson et al. (2007) mencionan que las comunidades forestales más impactadas son las que se desarrollan en los márgenes de los ríos; las cuales son asociaciones complejas y extremadamente frágiles (Meli et al. 2017), que son indispensables para mantener la conectividad entre poblaciones del ecosistema forestal (Granados-Sánchez et al. 2006).

De acuerdo con Santiago-Pérez et al. (2014), la vegetación asociada a la ribera de un río se le llama bosque de galería, que Rzedowski (1978) describió como agrupaciones arbóreas de hoja perenne y decidua, que se desarrollan a lo largo de corrientes de agua, con alturas de 4 hasta $40 \mathrm{~m}$, y se localizan en altitudes de 0 a $2800 \mathrm{~m}$, siendo Populus el género más representativo en las zonas áridas y semiáridas del norte del país. Mientras que Aguilar-Luna et al. (2018) mencionan que los bosques de galería ocupan franjas transversales a lo largo de las corrientes fluviales, con una estructura y función ecosistémica particular. Al mismo tiempo que son primordiales para mantener la calidad del agua y amortiguar los procesos de sedimentación de los lechos de los ríos (Granados-Sánchez et al. 2006), además, de proteger contra la erosión del suelo y proveer un hábitat para organismos acuáticos y terrestres (CamachoRico et al. 2006).

Aunque se han realizado estudios de la diversidad y estructura de los bosques de galería en México (Meli et al. 2017, Aguilar-Luna et al. 2018, CanizalesVelázquez et al. 2021), para el río Fuerte no se tiene registro. Al respecto la SEMARNAT (2014), indica que el estado cuenta con 3612.89 ha de bosques de galería en los municipios de Ahome, El Fuerte, Guasave y Sinaloa. Por lo que resulta prioritario analizar la diversidad de la vegetación del río Fuerte y generar información de los procesos ecológicos, para entender los patrones de la distribución actual. De acuerdo con Castillo-Castillo et al. (2017) el río Fuerte es la corriente hidrológica más importante de la parte norte del estado de Sinaloa y forma parte de la etnorregión Yoreme-Mayo (Sandoval-Forero y Meza-Hernández 2013), actualmente fragmentado territorialmente.

El Municipio de El Fuerte posee el 39.7\% (1 $434.19 \mathrm{ha}$ ) del bosque de galería en el norte de Sinaloa (SEMARNAT 2014). Sin embargo, presenta una tasa media anual de deforestación de $0.61 \%$, que lo sitúa entre los municipios más degradados del estado y del país (Monjardín-Armenta et al. 2017). Para Castellanos-Bolaños et al. (2010), la estructura arbórea es un indicador de la diversidad y la estabilidad ecológica de una población, esencial para entender la funcionalidad y el estado de conservación de los ecosistemas (Delgado-Zamora et al. 2016) que garantizan la provisión de bienes y servicios ambientales (Aguilar-Luna et al. 2018), como es la vegetación asociada a los recursos hidrológicos. Por lo anterior, el objetivo del presente trabajo fue evaluar y describir la diversidad y la estructura de la vegetación asociada al bosque de galería del río Fuerte, en el norte de Sinaloa. 


\section{MATERIALES Y MÉTODOS}

\section{Área de estudio}

El río Fuerte nace en la Sierra Madre Occidental, entre los estados de Chihuahua, Durango y Sonora; desemboca al norte del estado de Sinaloa, en el Golfo de California. La cuenca del río Fuerte se ubica en el noroeste de México, $25.68^{\circ}$ y $28.24^{\circ}$ LN, y $-106.12^{\circ}$ y $-109.43^{\circ}$ LO. Las localidades urbanas más importantes económica y demográficamente dentro de la cuenca son: Los Mochis, El Fuerte y Choix (Castillo-Castillo et al. 2017). La cuenca del río Fuerte es una de las más importantes dentro de la Región Hidrológica 10, es exorreica, con un área total de $36457 \mathrm{~km}^{2}$; el parteaguas presenta una elevación máxima de 3168 m s.n.m., el punto más bajo de la cuenca es de $-9 \mathrm{~m}$ s.n.m. Esta cuenca presenta una precipitación media anual de $691 \mathrm{~mm}$ y distribución temporal definida en dos periodos de lluvias en la zona, verano e invierno (Castillo-Castillo et al. 2017).

La sección de estudio, abarcó de la comunidad de San José de Cahuinahua, a la altura de la represa derivadora Cahuinahua hasta el ejido de Teroque Viejo. La ribera del cuerpo hídrico tiene una longitud de $19.53 \mathrm{~km}$, la vegetación asociada al margen que forma el polígono cuenta con una superficie de 540 ha. De las cuales 310 ha se ubican en el margen norte y 230 ha en el margen sur del río (Figura 1). Presenta un clima semiárido cálido con lluvias en verano $\left(B S 1\left(h^{\prime}\right) w\right)$, con una precipitación media de anual de $331 \mathrm{~mm}$ y $24^{\circ} \mathrm{C}$ de temperatura media anual. El uso del suelo predominante es agrícola, con algunos remanentes de matorral sarcocaule (SEMARNAT 2014).

\section{Diseño de muestreo}

Se utilizaron parcelas de muestreo circulares de $1000 \mathrm{~m}^{2}$ (Zacarias-Eslava et al. 2011, ChiquiniHeredia et al. 2017, Sánchez-Hernández et al. 2018), ya que de acuerdo con McRoberts et al. (2015) y Méndez-Osorio et al. (2018) requiere solo un punto de control, lo que las hace más confiables. Las parcelas se distribuyeron de forma aleatoria, 29 parcelas para el margen norte y 12 para el margen sur, con respecto al río. Las 41 parcelas constituyen una superficie total evaluada de 4.1 ha. Cada parcela fue georreferenciada (Figura 1) y dividida en unidades de muestreo para cada caracterización de los estratos arbóreos. Para árboles ( $\geq 7.5 \mathrm{~cm}$ de diámetro normal) la unidad de muestreo fue de $1000 \mathrm{~m}^{2}$. Una unidad de muestreo de $400 \mathrm{~m}^{2}$ para arbustos $(\geq 5 \mathrm{y} \leq 7.5 \mathrm{~cm}$ de diámetro normal). De $100 \mathrm{~m}^{2}$ para brinzales $(<5$ $\mathrm{cm}$ de diámetro normal) y por último, una unidad de muestreo de $4 \mathrm{~m}^{2}$ para plántulas $(<5 \mathrm{~cm}$ de diámetro normal) menores de $1.3 \mathrm{~m}$ de altura y mayores de 25 cm (Alanís-Rodríguez et al. 2015, García-García et al. 2019).

Se evaluaron las variables dasométricas de altura, el diámetro normal y la cobertura de la copa en sentido norte-sur y este-oeste. Con estos últimos datos se obtuvo el área de la copa y el área basal. Además se incluyeron datos ecológicos (uso del suelo, pendiente, exposición, perturbación y cobertura del suelo) y edáficos (compactación, textura y tipo de erosión) de cada parcela. Se realizó una fotocolecta con la totalidad de ejemplares registrados y se recolectaron tres muestras botánicas, las cuales se prensaron y depositaron físicamente en el laboratorio de ecología de la Universidad Autónoma Indígena de México. Para identificar las especies y su autoría se utilizaron claves taxonómicas (Pérez-Rodríguez 2008), bases de datos especializadas (The Plant List y Naturalista-CONABIO), estudios regionales de flora (Vega-Aviña et al. 1989, Gentry 1998) y el acervo virtual de la Red de Herbarios del noroeste de México.

\section{Análisis de la información}

Para describir la estructura de la vegetación arbórea del bosque de galería, de cada especie se determinó su abundancia de acuerdo con el número de individuos, su dominancia en función de su cobertura de copa y su frecuencia con base en su presencia en los sitios de muestreo (Albor-Pinto et al. 2017). Para lo cual se utilizó el Índice de Valor de Importancia (IVI), que sirvió para jerarquizar la importancia de cada especie en la vegetación, con base en la dominancia $\left(\mathrm{AR}_{i}\right)$, densidad $\left(\mathrm{DR}_{i}\right)$ y frecuencia relativa $\left(\mathrm{FR}_{i}\right)$ (Graciano-Ávila et al. 2018). 


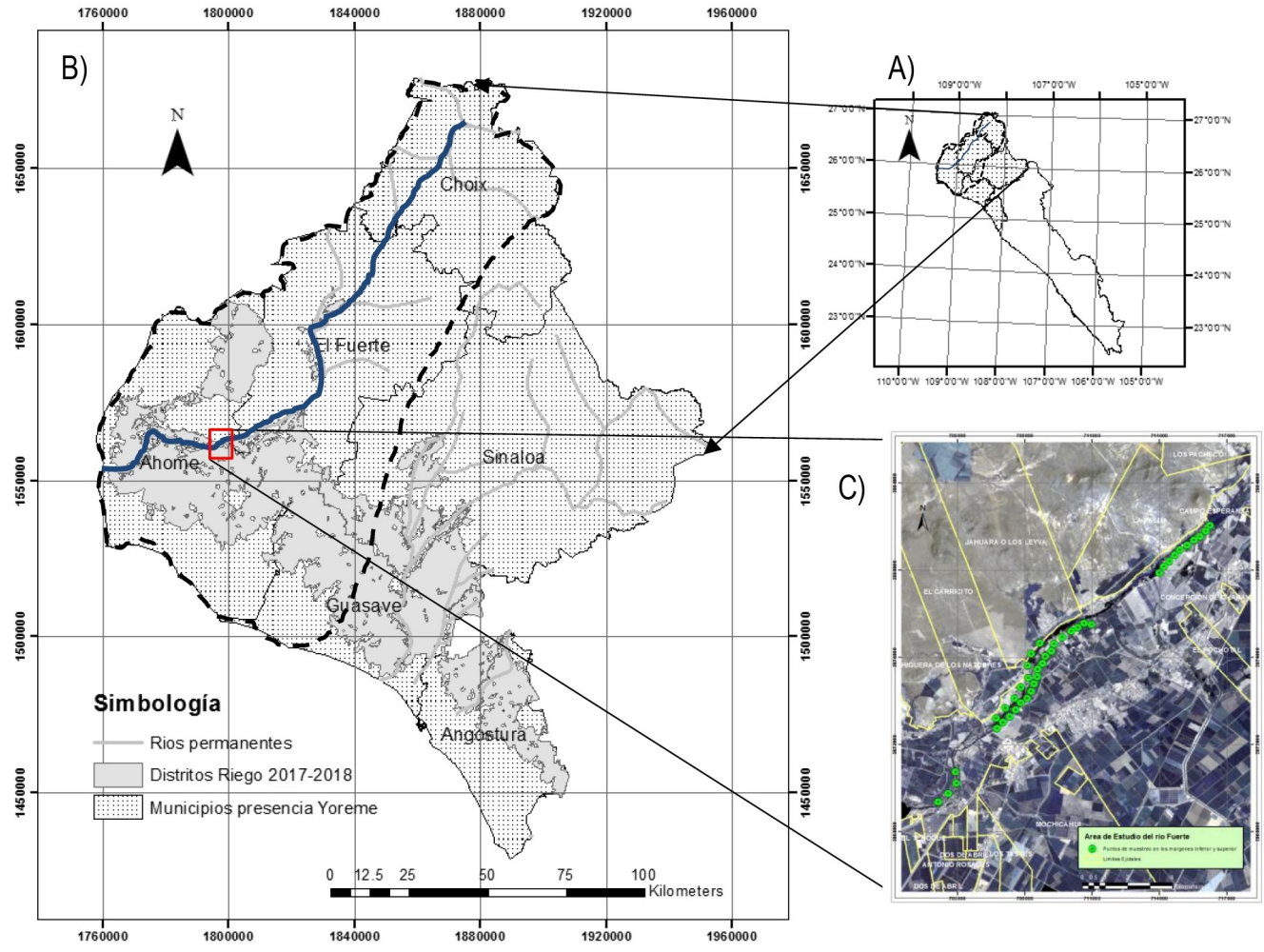

Figura 1. Mapa de ubicación del área de estudio. A) Mapa de Sinaloa indicando la cuenca del río Fuerte; B) Sección estudiada del río (rectángulo rojo) dentro de la cuenca del río Fuerte y los municipios que la conforman; C) Ubicación espacial de las parcelas de muestreo (círculos verdes) en los márgenes del río Fuerte.

$$
I V I=\frac{\sum_{n}^{i=1}\left(A R_{i}+D R_{i}+F R_{i}\right)}{3}
$$

Dónde: $\mathrm{AR}_{i}=$ abundancia de la especie $i$ respecto a la abundancia total, $\mathrm{DR}_{i}=$ dominancia relativa de la especie $i$ respecto a la dominancia total, y $\mathrm{FR}_{i}=$ frecuencia relativa de la especie $i$ respecto a la frecuencia total.

Para conocer la estructura horizontal de la vegetación, se agruparon los individuos medidos en categorías diamétricas como sigue: 5 (2.50 a 7.49 $\mathrm{cm}), 10(7.5 \mathrm{a} 12.49 \mathrm{~cm}), 15(12.5$ a $17.4 \mathrm{~cm}), 20$ (17.5 a $22.4 \mathrm{~cm}$ ), 25 (22.5 a $27.4 \mathrm{~cm}$ ), 30 (27.5 a 32.4 $\mathrm{cm}), 35(32.5$ a $37.4 \mathrm{~cm}), 40(37.5$ a $42.4 \mathrm{~cm}), 45$ $(42.5$ a $47.4 \mathrm{~cm}), 50(47.5$ a $52.4 \mathrm{~cm})$ y $65(62.5 \mathrm{a}$ $67.4 \mathrm{~cm}$ ). Para la estructura vertical se agruparon los individuos medidos en cinco categorías de altura, con intervalos de $5 \mathrm{~m}$ (Canizales-Velázquez et al. 2021).

Para determinar la diversidad $\alpha$ de las parcelas de muestreo del río Fuerte se utilizaron los índices de Margalef $\left(D_{M g}\right)$ que cuantifica el número de especies presentes, es decir la riqueza específica (AlanísRodríguez et al. 2015). El índice de Shannon-Wiener $\left(H^{\prime}\right)$ base logaritmo natural, el cual refiere a la estructura numérica de la comunidad, es decir, en la distribución proporcional de la abundancia de cada especie (Moreno 2001), que refleja la relación entre riqueza y uniformidad (Gutiérrez-Báez et al. 2012). El índice de Dominancia de Simpson $(\lambda)$, que mide la probabilidad de que dos individuos seleccionados al azar en las parcelas de muestreo sean de la misma especie (Sánchez-Hernández et al. 2018), proporcionando valores de dominancia de una especie (Gallardo-Cruz et al. 2005, García-García y SantosMoreno 2014). Finalmente el índice de Equidad $(E)$, que proporciona la diversidad observada respecto a la máxima diversidad esperada (Magurran 2003, Carreón-Sántos y Valdez-Hernández 2014). Las fórmulas empleadas fueron las siguientes:

$$
\text { Índice de Margalef: } D_{M g}=\frac{(S-1)}{\ln (N)}
$$


Dónde: $S=$ número de especies presentes, $N=$ número total de individuos.

Índice de Shannon-Wiener: $H^{\prime}=-\sum_{i=1}^{S} p_{i} \ln \left(p_{i}\right)$

$$
p_{i} \frac{n_{i}}{N}
$$

Dónde: $S=$ número de especies presentes, $\mathrm{p}_{i}=$ proporción de individuos de la especie $i, \mathrm{n}_{i}=$ número de individuos de la especie $i, N=$ número total de individuos.

Índice de Dominancia de Simpson: $\lambda=\sum_{n}^{i=1} p_{i}^{2}$

$$
p_{i} \frac{n_{i}}{N}
$$

Dónde: $\mathrm{p}_{i}=$ proporción de individuos de la especie $i$.

$$
\text { Índice de Equidad: } E=\frac{H^{\prime}}{\ln (S)}
$$

Dónde: $H^{\prime}=$ índice de Shannon-Wiener, $S$ = número de especies presentes.

\section{RESULTADOS}

\section{Composición florística}

Se registraron 27 especies (13 árboles, nueve arbustos y cinco hierbas) de las cuales 16 son nativas y 11 introducidas, todas estas especies tienen presencia en el margen sur, mientras que para el margen norte sólo se registraron 11 especies. La familia Fabaceae es la de mayor riqueza con 11 especies, seguida de Salicaceae con dos (Populus mexicana Moric y Salix bonplandiana H.B.K.), ambas presentes en los dos márgenes del río (Tabla 1).

\section{Estructura de la vegetación}

De acuerdo con los valores del IVI, en el bosque de galería del río Fuerte el $72.22 \%$ de la densidad es de árboles, el $18.58 \%$ de arbustos y el restante de hierbas. La copa de los árboles cubre más del $97 \%$ del área estudiada. El estrato arbóreo tuvo un valor de importancia ecológico del $79.18 \%$, mientras que para el arbustivo fue de 14.11\% (Tabla
2). El $85 \%$ del valor de importancia fue para especies nativas (Tabla 2).

Las familias Fabaceae (50.27\%) y Salicaceae Mirb. (30.55\%) representaron más del $80 \%$ del valor de importancia ecológica de la vegetación del río Fuerte (Figura 2). El área evaluada presentó una densidad de 145 individuos ha-1 y una cobertura de copas de $6184.44 \mathrm{~m}^{2} \mathrm{ha}^{-1}$. A nivel de especie, Populus mexicana, Pithecellobium dulce (Roxb.) Benth. y Prosopis juliflora (Sw.) DC fueron las más sobresalientes según los valores del IVI, pues representan el $61.71 \%$ del total de la comunidad. El $44.45 \%$ de las especies presentan valores de IVI inferiores al 1.0\% (Tabla 3).

La estructura de la vegetación presentó un patrón de distribución en donde el número de individuos fue inversamente proporcional a las categorías diamétricas y de altura. Además, presentó una estructura vertical con diámetros bajos, en una hectárea más del $60 \%$ de la vegetación se encontró en la categoría diamétrica de cinco, aunque fue posible encontrar cinco árboles con diámetros mayores a $40 \mathrm{~cm}$ (Figura 3). La estructura vertical de la vegetación fue baja, la mayor densidad de individuos (74\%) presentó alturas inferiores a $12.5 \mathrm{~m}$, pero en una hectárea fue posible encontrar nueve árboles con altura superior a los $20 \mathrm{~m}$ (Figura 4).

\section{Diversidad de la vegetación}

Se estimaron los índices de diversidad para las unidades de muestreo y se caracterizaron los estratos de la vegetación del río. De acuerdo al índice de Margalef, la mayor riqueza se dio en el estrato arbóreo (1.61) y en la regeneración de plántulas (2.3), mientras que los brinzales (0.8) presentaron la menor riqueza. Para la diversidad, el índice de Shannon-Wiener el estrato de plántulas (1.99) presentó la mayor diversidad. De acuerdo con los valores del índice de Simpson, se muestra que los estratos de plántulas $(0.17)$ y arbóreo $(0.28)$, se encontraron dominados por pocas especies y los brinzales (0.47) presentaron homogeneidad en el número de ellas. En los estratos de árbol, arbusto, brinzal y plántula las especies se presentaron de manera uniforme, de acuerdo con el índice de Equidad (Tabla 4). 
Tabla 1. Lista de especies que forman parte de la vegetación del bosque de galería en el río Fuerte.

\begin{tabular}{|c|c|c|c|c|c|}
\hline Número de Colecta * & Especie & Nombre común & Familia & Presencia & Forma biológica \\
\hline 21-uaim & Acacia cochliacantha Humb. \& Bonpl. ex Willd & Huinolo & Fabaceae & Nativa & Árbol \\
\hline 02-uaim & Acacia farnesiana $(\mathrm{L})$ Willd. & Vinorama & Fabaceae & Nativa & Árbol \\
\hline 12-uaim & Albizia sinaloensis Britton \& Rose & Palo joso & Fabaceae & Nativa & Árbol \\
\hline 33-uaim & Caesalpinia cacalaco Humb. \& Bonpl. & Huizache & Fabaceae & Nativa & Árbol \\
\hline 41-uaim & Crataeva tapia $\mathrm{L}$ & Perihuete & Capparaceae & Nativa & Árbol \\
\hline 08-uaim & Guazuma ulmifolia Lam & Guácima & Sterculiaceae & Nativa & Árbol \\
\hline 01 -uaim & Leucaena leucocephala (Lam.) de wit. & Guaje & Fabaceae & Nativa & Árbol \\
\hline 20-uaim & Parkinsonia aculeata L & Palo verde & Fabaceae & Nativa & Árbol \\
\hline 13-uaim & Parkinsonia praecox (Ruiz \& Pav.) Hawkins & Palo brea & Fabaceae & Nativa & Árbol \\
\hline 10-uaim & Pithecellobium dulce (Roxb.) Benth. & Guamúchil & Fabaceae & Nativa & Árbol \\
\hline 25-uaim & Populus mexicana Moric & Álamo & Salicaceae & Nativa & Árbol \\
\hline 17-uaim & Prosopis juliflora (Sw.) DC & Mezquite & Fabaceae & Nativa & Árbol \\
\hline 19-uaim & Salix bonplandiana H.B.K & Sauce & Salicaceae & Introducida & Árbol \\
\hline 29-uaim & Abutilon abutiloides (Jacq.) & Malva & Malvaceae & Nativa & Arbusto \\
\hline 11-uaim & Crotalaria incana $\mathrm{L}$ & Cascabelillo & Fabaceae & Introducida & Arbusto \\
\hline 15-uaim & Cryptostegia grandiflora Roxb. Ex R.Br & Chicote & Apocynaceae & Introducida & Arbusto \\
\hline 24-uaim & Franseria ambrosioides & Chicura & Asteraceae & Nativa & Arbusto \\
\hline 31-uaim & Mascagnia macroptera (Sesse \& Moc.) Niedenzu & Matanene & Malpighiaceae & Nativa & arbusto \\
\hline 35-uaim & Pisonia capitata (S. Watson) Standl & Garambullo & Nyctaginaceae & Introducida & Arbusto \\
\hline 39-uaim & Ricinus communis $\mathrm{L}$ & Higuerilla & Euphorbiaceae & Introducida & Arbusto \\
\hline 42-uaim & Vallesia glabra (Cav.) Link & Cacaragua & Apocynaceae & Nativa & Arbusto \\
\hline 14-uaim & Argemone ochroleuca Sweet & Cardo santo & Papaveraceae & Introducida & Hierba \\
\hline 30-uaim & Arundo donax $\mathrm{L}$ & Carrizo & Poaceae & Introducida & Hierba \\
\hline 44-uaim & Leonotis nepetifolia (L.) R. Br & Cola de león & Lamiaceae & Introducida & Hierba \\
\hline 05-uaim & Mimosa pudica L & Cuatante & Fabaceae & Introducida & Hierba \\
\hline 38-uaim & Momordica charantia L & Pepinillo del monte & Cucurbitaceae & Introducida & Hierba \\
\hline 43-uaim & Nicotiana glauca Graham & Tabaco silvestre & Solanaceae & Introducida & Hierba \\
\hline
\end{tabular}

Tabla 2. Abundancia, dominancia, frecuencia y el índice de valor de importancia (IVI) de las especies nativas e introducidas y las formas biológicas del bosque de galería en el río Fuerte.

\begin{tabular}{lrrrr}
\hline & Abundancia & Dominancia & Frecuencia & IVI \\
\hline Nativos & 83.11 & 95.71 & 78.83 & 85.88 \\
Introducidos & 16.89 & 4.29 & 21.17 & 14.12 \\
$\quad$ Total & 100.00 & 100.00 & 100.00 & 100.00 \\
Árboles & 72.22 & 97.07 & 68.24 & 79.18 \\
Arbustos & 18.58 & 1.66 & 22.09 & 14.11 \\
Hierbas & 9.20 & 1.27 & 9.67 & 6.71 \\
\multicolumn{1}{r}{ Total } & 100.00 & 100.00 & 100.00 & 100.00 \\
\hline
\end{tabular}

\section{DISCUSIÓN}

\section{Composición florística}

La vegetación del río Fuerte está compuesta por 27 especies, lo que es similar a lo encontrado por Scott et al. (2009) que reportó 27 y 31 especies arbóreas para el río Cuchujaqui y el río Mayo en Sonora. Estos ríos tienen cuencas adyacentes al río Fuerte, presentan condiciones ambientales similares y con vegetación semiárida. Cabe mencionar que su estudio fue realizado utilizando imágenes satelitales, con lo que registró 79 especies para toda la vegetación ribereña del estado de Sonora. Por su parte Solis-Garza et al. (2017) reportaron 77 especies para los ríos Sonora y Bacanuchi, ubicados en la zona centro del estado de Sonora. Para el caso de bosques de galería en zonas semiáridas de la parte noreste de México, Treviño-Garza et al. (2001) reportaron 19 especies de la vegetación de los ríos Cabezones en el centro sur de Nuevo León. Mientras que Treviño-Garza et al. (2001) y CanizalesVelázquez et al. (2010) mencionan que la estructura de la vegetación del río Ramos en Nuevo León, se integra por siete especies, lo que contrasta con la vegetación ribereña de la zona noroeste del país que presenta una mayor composición de especies. 


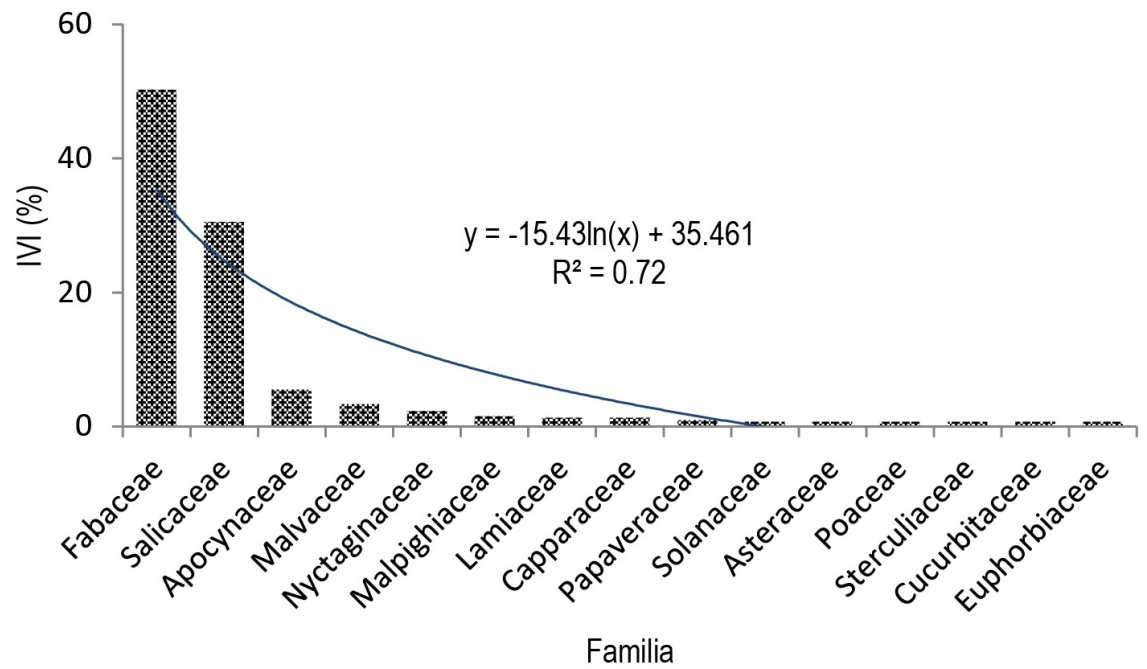

Figura 2. Índice de valor de importancia (IVI) de las familias de la vegetación del bosque de galería en el río Fuerte. La línea de tendencia se presenta en color azul.

Tabla 3. Atributos estructurales y el valor de importancia ecológica de las especies registradas en el río Fuerte.

\begin{tabular}{|c|c|c|c|c|c|c|c|}
\hline \multirow[b]{2}{*}{ Especies } & \multicolumn{2}{|c|}{ Abundancia } & \multicolumn{2}{|c|}{ Dominancia } & \multicolumn{2}{|c|}{ Frecuencia } & \multirow[b]{2}{*}{$\begin{array}{l}\text { IVI } \\
(\%)\end{array}$} \\
\hline & $\begin{array}{l}\text { Absoluta } \\
\left(\mathrm{N} / \mathrm{ha}^{-1}\right)\end{array}$ & $\begin{array}{r}\text { Relativa } \\
(\%)\end{array}$ & $\begin{array}{r}\text { Absoluta } \\
\left(\mathrm{m}^{2} / \mathrm{ha}^{-1}\right)\end{array}$ & $\begin{array}{r}\text { Relativa } \\
(\%)\end{array}$ & Absoluta & $\begin{array}{r}\text { Relativa } \\
(\%)\end{array}$ & \\
\hline Populus mexicana & 20.17 & 13.91 & 3293.37 & 53.25 & 65.08 & 17.20 & 28.12 \\
\hline Pithecellobium dulce & 44.49 & 30.68 & 1633.74 & 26.42 & 47.69 & 12.60 & 23.23 \\
\hline Prosopis juliflora & 14.52 & 10.01 & 540.68 & 8.74 & 46.69 & 12.34 & 10.36 \\
\hline Parkinsonia aculeata & 7.15 & 4.93 & 171.05 & 2.77 & 29.74 & 7.86 & 5.19 \\
\hline Acacia farnesiana & 7.95 & 5.48 & 86.69 & 1.40 & 23.84 & 6.30 & 4.39 \\
\hline Vallesia glabra & 6.63 & 4.57 & 41.48 & 0.67 & 22.12 & 5.85 & 3.70 \\
\hline Abutilon abutiloides & 9.58 & 6.61 & 0.78 & 0.01 & 12.78 & 3.38 & 3.33 \\
\hline Mimosa pudica & 3.36 & 2.32 & 76.10 & 1.23 & 19.39 & 5.12 & 2.89 \\
\hline Salix bonplandiana & 4.00 & 2.76 & 127.74 & 2.07 & 9.33 & 2.47 & 2.43 \\
\hline Pisonia capitata & 2.41 & 1.66 & 44.08 & 0.71 & 17.24 & 4.56 & 2.31 \\
\hline Cryptostegia grandiflora & 3.73 & 2.57 & 14.80 & 0.24 & 10.05 & 2.66 & 1.82 \\
\hline Mascagnia macroptera & 2.75 & 1.90 & 0.83 & 0.01 & 10.34 & 2.73 & 1.55 \\
\hline Albizia sinaloensis & 0.93 & 0.64 & 75.01 & 1.21 & 9.33 & 2.47 & 1.44 \\
\hline Leonotis nepetifolia & 4.48 & 3.09 & 0.42 & 0.01 & 3.44 & 0.91 & 1.34 \\
\hline Crataeva tapia & 1.76 & 1.21 & 16.02 & 0.26 & 9.33 & 2.47 & 1.31 \\
\hline Argemone ochroleuca & 2.75 & 1.90 & 0.18 & 0.00 & 3.44 & 0.91 & 0.94 \\
\hline Parkinsonia praecox & 1.37 & 0.94 & 33.96 & 0.55 & 3.44 & 0.91 & 0.80 \\
\hline Caesalpinia cacalaco & 1.03 & 0.71 & 20.91 & 0.34 & 3.44 & 0.91 & 0.65 \\
\hline Nicotiana glauca & 1.37 & 0.94 & 1.68 & 0.03 & 3.44 & 0.91 & 0.63 \\
\hline Franseria ambrosioides & 0.83 & 0.57 & 0.62 & 0.01 & 4.16 & 1.10 & 0.56 \\
\hline Arundo donax & 1.03 & 0.71 & 0.08 & 0.00 & 3.44 & 0.91 & 0.54 \\
\hline Leucaena leucocephala & 0.68 & 0.47 & 0.36 & 0.01 & 3.44 & 0.91 & 0.46 \\
\hline Crotalaria incana & 0.68 & 0.47 & 0.03 & 0.00 & 3.44 & 0.91 & 0.46 \\
\hline Guazuma ulmifolia & 0.34 & 0.23 & 3.31 & 0.05 & 3.44 & 0.91 & 0.40 \\
\hline Momordica charantia & 0.34 & 0.23 & 0.24 & 0.00 & 3.44 & 0.91 & 0.38 \\
\hline Acacia cochliacantha & 0.34 & 0.23 & 0.15 & 0.00 & 3.44 & 0.91 & 0.38 \\
\hline Ricinus communis & 0.34 & 0.23 & 0.13 & 0.00 & 3.44 & 0.91 & 0.38 \\
\hline Total & 145.01 & 100.00 & 6184.44 & 100.00 & 378.39 & 100.00 & 100.00 \\
\hline
\end{tabular}

IVI: Índice de Valor de Importancia.

La familia botánica mejor representada fue la Fabaceae con 11 especies, que de acuerdo con
Rzedowski (1978) es la más representativa en los ecosistemas ribereños de zonas semiáridas. Esta 


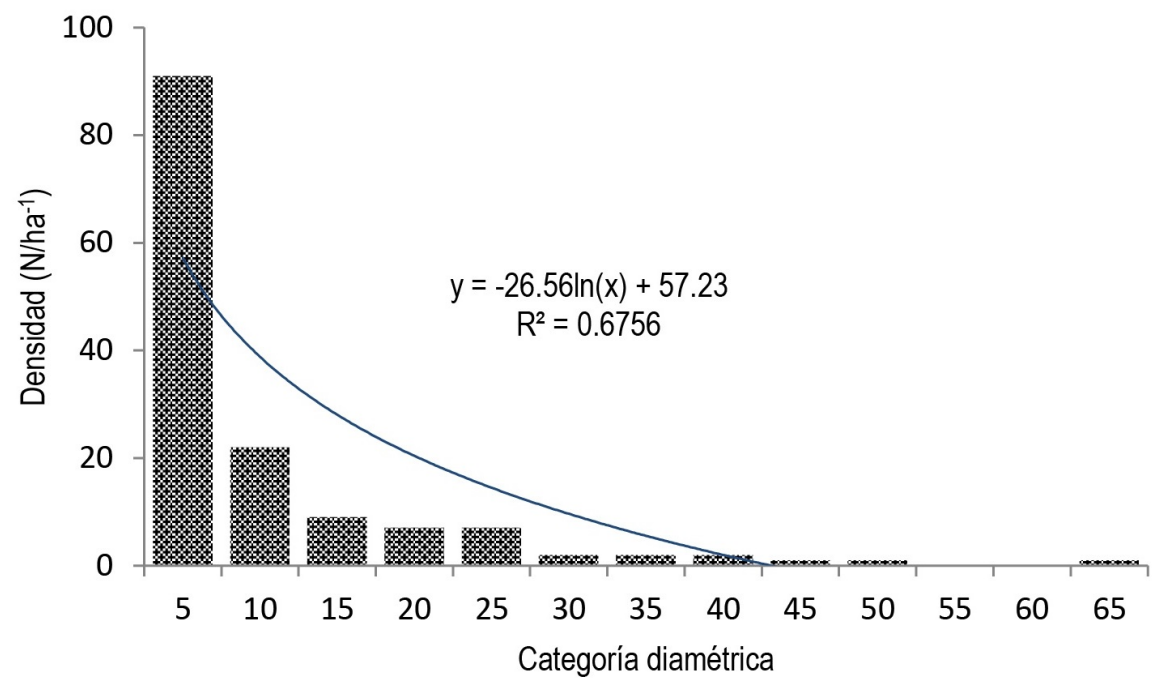

Figura 3. Distribución de categorías diamétricas para la vegetación arbórea del bosque de galería en el río Fuerte. Se presenta la línea de tendencia.

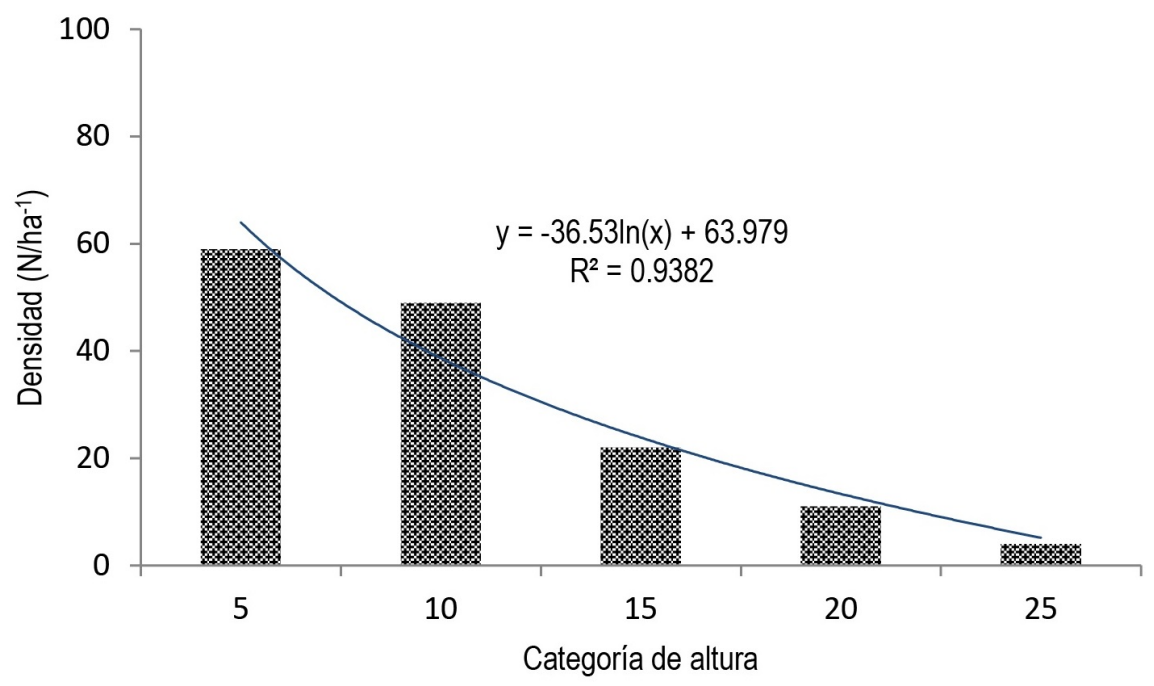

Figura 4. Distribución de las categorías de altura para la vegetación arbórea del bosque de galería en el río Fuerte. Se presenta línea de tendencia central.

Tabla 4. Valores de los índices de Equidad, Margalef, Simpson y Shanon-Wiener para los estratos de la vegetación del río Fuerte.

\begin{tabular}{lccccc}
\hline Índices de diversidad alfa & Árbol & Arbusto & Brinzal & Plántula & Promedio \\
\hline Índice de Equidad & 0.73 & 0.77 & 0.76 & 0.85 & 0.77 \\
Índice de Margalef & 1.61 & 1.07 & 0.80 & 2.30 & 1.44 \\
Índice de Simpson & 0.28 & 0.39 & 0.47 & 0.17 & 0.32 \\
Índice de Shannon-Wiener & 1.50 & 1.16 & 1.03 & 1.99 & 1.42 \\
\hline
\end{tabular}

situación también coincide con lo reportado por Scott et al. (2009) y Solís-Garza et al. (2017), pues 22 especies de la vegetación ribereña de Sonora pertenecen a esta familia. Mientras que, en Nuevo
León, Treviño-Garza et al. (2001) reportan cuatro especies en el río Cabezones y dos para el río Ramos (Canizales-Velázquez et al. 2010) de la misma familia. En tanto en el río Camachito reportan a la 
familia Fabaceae con el mayor número de especies con siete, seguida por la familia Salicaceae con dos (Canizales-Velázquez et al. 2021).

Los problemas que impactan la vegetación del río Fuerte son la ganadería extensiva, la actividad recreativa, la tala y extracción de materiales para construcción, que contaminan con desechos. Estas causas perturban el hábitat, provocando la mortandad de plántulas y evitando la regeneración, siendo similar a lo que ocurre con la vegetación de los ríos en el estado de Sonora (Scott et al. 2009, Solís-Garza et al. 2017) y del estado de Nuevo León (TreviñoGarza et al. 2001, Canizales-Velázquez et al. 2010). Otra situación hallada en el río Fuerte, es que el $40 \%$ de las especies son introducidas, valor similar al hallado por Scott et al. (2009) y Solís-Garza et al. (2017) para la vegetación ribereña de Sonora (25 y $34 \%)$. Esto se explica también por los disturbios ocasionados por la actividad humana. Según MéndezToribio et al. (2014) las perturbaciones aumentan el número de especies, al incorporar especies exóticas. Aunque puede ocurrir lo contrario, que el impacto antropogénico disminuya la riqueza florística de los ecosistemas ribereños. La riqueza en la composición florística de los ríos sin duda, es un indicador de la estabilidad ecológica, lo que garantiza la provisión de bienes y servicios ambientales a las comunidades ribereñas (Castellanos-Bolaños et al. 2010, AguilarLuna et al. 2018).

\section{Estructura de la vegetación}

El estrato arbóreo tuvo un valor de importancia ecológica del $79.18 \%$, mientras que el arbustivo fue de $14.11 \%$. Valores similares a lo reportado por Aguilar-Luna et al. (2018) con 76\% para el estrato arbóreo del río Xaltatempa, en Puebla, y también similar a lo encontrado por Scott et al. (2009) quienes agruparon el estrato arbóreo (70\%) y arbustivo (30\%) para la vegetación ribereña del estado de Sonora. Aunque contrario a lo registrado por Solís-Garza et al. (2017) en los ríos Sonora y Bacanuchi, donde los arbustos fueron la forma de crecimiento predominante con el $50.6 \%$ y los árboles la tercera con valor de $18.2 \%$. El autor lo atribuye a los disturbios y la fragmentación del suelo para uso agropecuario, como de los problemas ocasionados por la contaminación e impactos de la minería. Por lo anterior, la vegetación de ambos ríos está en constante sucesión secundaria.

De acuerdo con el índice de valor de importancia, las especie $P$. mexicana, $P$. dulce y $P$. juliflora representaron el $61.71 \%$ del total de la comunidad, lo que coincide con Solís-Garza et al. (2017), ya que Populus fremontii S. Watson, Prosopis velutina Wooton y Salix gooddingii C.R.Ball alcanzaron el $69.8 \%$; y similar a lo encontrado por Scott et al. (2009), donde estas especies representan el $56 \%$ de la vegetación ribereña en Sonora. En esta zona del noroeste del país, los géneros Populus L., Prosopis L. Salix L y Taxodium Rich. son los más representativos del bosque de galería. Al respecto, GranadosSánchez et al. (2006) sostienen que estos géneros constituyen los elementos principales de la estructura de los bosques de galería del país.

La vegetación del río Fuerte presentó en su mayoría diámetros en la categoría de 5 (60\%), con alturas promedio de $10 \mathrm{~m}$, ésta estructura de la vegetación presentó un patrón de distribución donde el número de individuos fue inversamente proporcional a las categorías de diámetro y de altura. De acuerdo con Solís-Garza et al. (2017), cuando los diámetros son pequeños la comunidad vegetal puede considerarse juvenil. Este crecimiento de la vegetación se debe a los disturbios que ocasionan las actividades antropogénicas, además de la crecida anual del río, por lo que el ecosistema ribereño es dinámico y sucesional (Aguilar-Luna et al. 2018). La falta de establecimiento y crecimiento de nuevos individuos, estaría afectando el remplazo de individuos longevos (con énfasis en Populus mexicana) que experimentan mortandad o tala en sus poblaciones.

\section{Diversidad de la vegetación}

La mayor diversidad la presentó el estrato de plántulas, este dato puede deberse a los constantes disturbios que han modificado el uso del suelo del ecosistema ribereño, lo que da lugar a que ocurra la regeneración de las especies que compiten para asegurar su supervivencia. En general, los valores de los índices de Shannon-Wiener y de Simpson indicaron 
una diversidad alta de la vegetación del río Fuerte, aunque solamente tres especies proporcionan el 55\% de la abundancia relativa, cuyo valor coincide con lo reportado por Solís-Garza et al. (2017) para los ríos Bacanuchi y Sonora. En condiciones ambientales similares, Canizales-Velázquez et al. (2010) reportaron valores muy inferiores (de 0.18 a 0.74 ) para la vegetación del río Ramones, en Nuevo León. Por su parte Aguilar-Luna et al. (2018), encontraron valores (0.54) casi tres veces menores en el río Xaltatempa, en Puebla. Estos valores tan bajos pueden atribuirse a que la vegetación de estos ríos está compuesta por pocas especies y sólo una de ellas tiene mayor frecuencia. Este hallazgo confirma que el valor de los índices de diversidad se puede mejorar con la protección de los ecosistemas ribereños.

\section{CONCLUSIONES}

La vegetación del río Fuerte presentó una composición florística de 27 especies, de las cuales más de la mitad son nativas y tres son géneros arbóreos abundantes (de relevancia cultural), indicando una diversidad alta. La familia botánica con mayor importancia ecológica es la Fabaceae. La estructura arbórea presentó la mayor importancia ecológica con diámetros en la clase de 5 y con altura promedio de $10 \mathrm{~m}$. No obstante, la mayor diversidad se presentó en el estrato de plántulas, el cual, sufre mortandad y no se regenera afectando la dinámica de las poblaciones. Estos datos y la presencia de especies exóticas son indicativos de un ecosistema con presencia de disturbios, cuya funcionalidad está siendo modificada por las actividades agropecuarias, como por acciones de las poblaciones ribereñas en este importante cuerpo de agua regional.

\section{AGRADECIMIENTOS}

A la Universidad Autónoma Indígena de México por la vinculación y su papel en la educación regional. La investigación se realizó con Fondos SEPCONACYT "Patrimonio biocultural de los Yoremes y Yoris del norte de Sinaloa": Clave: 243129. Proyecto apoyado por el Fondo Sectorial de Investigación para la Educación.

\section{LITERATURA CITADA}

Aguilar-Luna JMA, Loeza-Corte JM, García-Villanueva E, Hernández-Fernández LA (2018) Arboreal vegetation structure and diversity in the galery forest of the Xaltatempa river, Puebla, Mexico. Madera y Bosques 24: $1-11$.

Alanís-Rodríguez E, Jiménez-Pérez J, Mora-Olivo A, Martínez-Ávalos JG, Mata-Balderas JM, Chávez-Costa AC, Rubio-Camacho EA (2015) Estructura y diversidad del matorral submontano contiguo al área metropolitana de Monterrey, Nuevo León, México. Acta Botánica Mexicana 113: 1-19.

Albor-Pinto C, Tun-Garrido J, Ortiz-Díaz JJ (2017) Diversidad, estructura y afinidades florísticas de un bosque temporalmente inundable de la Península de Yucatán. Revista de Biología Tropical 65: 868-880.

Camacho-Rico F, Trejo I, Bonfil C (2006) Estructura y composición de la vegetación ribereña de la Barranca del río Tembembe, Morelos, México. Boletín de la Sociedad Botánica de México 78: 17-31.

Canizales-Velázquez PA, Alanís-Flores GJ, Favela-Lara S, Torres-Morales M, Alanís-Rodríguez E, JiménezPérez J, Padilla-Rangel H (2010) Efecto de la actividad turística en la diversidad y estructura del bosque de galería en el noreste de México. Ciencia UANL 13: 55-63.

Canizalez-Velázquez PA, Alanís-Rodríguez E, García-García SA, Olguín-Estrada VA, Collantes-Chávez-Costa A (2021) Estructura y diversidad arbórea de un bosque de galería urbano en el río Camachito, noreste de México. Polibotánica 51: 91-105. 
Carreón-Santos RJ, Valdez-Hernández Jl (2014) Estructura y diversidad arbórea de vegetación secundaria derivada de una selva mediana subperennifolia en Quintana Roo. Revista Chapingo Serie Ciencias Forestales y del Ambiente 20: 119-130.

Castellanos-Bolaños JF, Treviño-Garza EJ, Aguirre-Calderón OA, Jiménez-Pérez J, Velázquez-Martínez A (2010) Diversidad arbórea y estructura espacial de bosques de pino-encino en Ixtlán de Juárez, Oaxaca. Revista Mexicana de Ciencias Forestales 1: 39-52.

Castillo-Castillo M, Ibáñez-Castillo LA, Valdez JB, Arteaga-Ramírez R, Vázquez-Peña MA (2017) Análisis de sequías meteorológicas en la cuenca del río Fuerte, México. Tecnología y Ciencias del Agua 8: 35-52.

Chiquini-Heredia W, Esparza-Olguín L, Peña-Ramírez Y, Maya-Martínez A, Martínez-Romero E (2017) Estructura y diversidad en selva inundable al centro y sur de Calakmul. Ecosistemas y Recursos Agropecuarios 4: 511-524.

Delgado-Zamora DA, Heynes-Silerio SA, Mares-Quiñones MD, Piedra-Leandro NL, Retana-Rentería FI, Rodríguez-Corral K, Villanueva-Hernández Al, González-Elizondo MS, Ruacho-González L (2016) Diversidad y estructura arbórea de dos rodales en Pueblo Nuevo, Durango. Revista Mexicana de Ciencias Forestales 7: 94-107.

Gallardo-Cruz JA, Meave JA, Pérez-García EA (2005) Estructura, composición y diversidad de la selva baja caducifolia del Cerro Verde, Nizanda (Oaxaca), México. Boletín de la Sociedad Botánica de México 76: 19-35.

García-García SA, Narváez-Flores R, Olivas-García JM, Hernández-Salas J (2019) Diversidad y estructura vertical del bosque de pino-encino en Guadalupe y Calvo, Chihuahua. Revista Mexicana de Ciencias Forestales 10: 41-63.

García-García JL, Santos-Moreno A (2014) Efectos de la estructura del paisaje y de la vegetación en la diversidad de murciélagos filostómidos (Chiroptera: Phyllostomidae) de Oaxaca, México. Revista de Biología Tropical 62: 217-239.

Gentry HS (1998) Gentry's Rio Mayo plants: The tropical deciduous forest and environs of Northwest Mexico. University of Arizona Press. Arizona, United States. 558p.

Graciano-Ávila G, Alanís-Rodríguez E, Aguirre-Calderón ÓA, Rubio-Camacho EA, González-Tagle MA (2018) Estructura y diversidad postincendio en un área del matorral espinoso tamaulipeco. Polibotánica 45: 89100.

Granados-Sánchez D, Hernández-García MA, López-Ríos GF (2006) Ecología de las zonas ribereñas. Revista. Chapingo Serie Ciencias Forestales y del Ambiente 12: 55-69.

Gutiérrez-Báez C, Ortiz-Díaz JJ, Flores-Guido JS, Zamora-Crescencio P (2012) Diversidad, estructura y composición de las especies leñosas de la selva mediana subcaducifolia del punto de unión territorial (Put) de Yucatán, México. Polibotánica 33: 151-174.

INEGI (2014) Conjunto de datos vectoriales edafológicos, escala 1: 250000 Serie IV; continuo nacional, escala: 1: 250 000. Ed. 2. Instituto Nacional de Estadística, Geografía e Informática. Aguascalientes, México.

Magurran AE (2003) Measuring biological diversity. Oxford, England: Blackwell Publishing. New Jersey, United States. 264p.

McRoberts RE, Tomppo EO, Czaplewski EL (2015) Diseños de muestreo de las evaluaciones forestales nacionales. SLU. 1. FAO. Roma, Italia. 21p. 
Meli P, Ruiz L, Aguilar R, Rabasa A, Rey Benayas JM, Carabias J (2017) Bosques ribereños del trópico húmedo de México: un caso de estudio y aspectos críticos para una restauración exitosa. Madera y Bosques 23 : 181-193.

Méndez-Osorio C, Mora-Donjuán CA, Alanís-Rodríguez E, Jiménez-Pérez J, Aguirre-Calderón OA, TreviñoGarza EJ, Pequeño-Ledezma MÁ (2018) Fitodiversidad y estructura de un bosque de pino-encino en la Sierra Madre del Sur, México. Revista Mexicana de Ciencias Forestales 9: 35-53.

Méndez-Toribio M, Zermeño-Hernández I, Ibarra-Manríquez G (2014) Effect of land use on the structure and diversity of riparian vegetation in the Duero river watershed in Michoacán, Mexico. Plant Ecology 215: 285-296.

Monjardín-Armenta SA, Pacheco-Angulo CE, Plata-Rocha W, Corrales-Barraza G (2017) La deforestación y sus factores causales en el estado de Sinaloa, México. Madera y Bosques 23: 7-22.

Moreno CE (2001) Métodos para medir la biodiversidad. M\&T-Manuales y Tesis SEA. Vol. 1. Zaragoza, España. $84 \mathrm{p}$.

Pérez-Rodríguez PM (2008) Claves de determinación botánica: Con énfasis en familias de árboles. Universidad Autónoma Chapingo. Libro universitario. Estado de México, México. 307p.

Richardson DM, Holmes PM, Esler KJ, Galatowitsch SM, Stromberg JC, Kirkman SP, Pyšek P, Hobbs RJ (2007) Riparian vegetation: degradation, alien plant invasions, and restoration prospects. Diversity Distribution 13: 126-139.

Rzedowski J (1978) Vegetación acuática y subacuática. En: Vegetación de México. Editorial Limusa, Distrito Federal, México. 364p.

Sánchez-Hernández MA, Fierros-González AM, Velázquez-Martínez A, De los Santos-Posadas HM, Aldrete A, Cortés-Díaz E (2018) Estructura, riqueza y diversidad de especies de árboles en un bosque tropical caducifolio de Morelos. Revista Mexicana de Ciencias Forestales 9: 131-156.

Sandoval-Forero EA, Meza-Hernández ME (2013) La interculturalidad en la etnorregión Yoreme Mayo de Sinaloa. Papeles de Población 19: 193-204.

Santiago-Pérez AL, Ayón-Escobedo A, Rosas-Espinoza VC, Rodríguez-Zaragoza FA, Toledo-González SL (2014) Estructura del bosque templado de galería en la Sierra de Quila, Jalisco. Revista Mexicana de Ciencias Forestales 5: 144-159.

Scott ML, Nagler PL, Glenn EP, Valdes-Casillas C, Erker JA, Reynolds EW, Shafroth PB, Gomez-Limon E, Jones CL (2009) Assessing the extent and diversity of riparian ecosystems in Sonora, Mexico. Biodiversity and Conservation 18: 247-269.

SEMARNAT (2014) Inventario estatal forestal y de suelos-Sinaloa 2014. Colección de inventarios estatales forestales y de suelos 2013-2014. Secretaría de Medio Ambiente y Recursos Naturales. Comisión Nacional Forestal. México. 161p.

Solís-Garza G, Robles-López H, Castellanos-Villegas AE (2017) Estructura y composición de la vegetación ribereña en zonas áridas. El caso de los ríos Bacanuchi y Sonora en el noroeste de México. Biotécnica 19: 3-12.

Treviño-Garza EJ, Cavazos-Camacho C, Aguirre-Caldrón OA (2001) Distribución y estructura de los bosques de galería en dos ríos del centro sur de Nuevo León. Madera y Bosques 7: 13-25.

Vega-Aviña R, Bojórquez G, Hernández-Álvarez F (1989) Flora de Sinaloa. Universidad Autónoma de Sinaloa. Sinaloa, México. 49p. 
Villaseñor JL, Ortíz E (2014) Biodiversidad de las plantas con flores (División Magnoliophyta) en México. Revista Mexicana de Biodiversidad 85: 134-142.

Zacarias-Eslava LE, Cornejo-Tenorio G, Cortés-Flores J, González-Castañeda N, Ibarra-Manríquez G (2011) Composición, estructura y diversidad del cerro El Águila, Michoacán, México. Revista Mexicana de Biodiversidad 82: 854-869. 